\title{
Studying the interactions in a mammalian nerve fiber: a functional modeling approach
}

\author{
Vijay Sadashivaiah, Pierre Sacré, Yun Guan, William S. Anderson, and Sridevi V. Sarma, IEEE Member
}

\begin{abstract}
Modern therapeutic interventions are increasingly favoring electrical stimulation to treat neurophysiological disorders. These therapies are associated with suboptimal efficacy since most neurostimulation devices operate in an open-loop manner (i.e., stimulation settings like frequency, amplitude are preprogrammed). A closed-loop system can dynamically adjust stimulation parameters and may provide efficient therapies. Computational models used to design these systems vary in complexity which can adversely affect their real-time performance. In this study, we compare two models of varying degrees of complexity. We constructed two computational models of a myelinated nerve fiber (functional versus mechanistic) each receiving two inputs: the underlying physiological activity at one end of the fiber, and the external stimulus applied to the middle of the fiber. We then defined relay reliability as the percentage of physiological action potentials that make it to the other end of the nerve fiber. We applied the two inputs to the fiber at various frequencies and analyze reliability. We found that the functional model and the mechanistic model have similar reliability properties, but the functional model significantly decreases the computational complexity and simulation run time. This modeling effort is the first step towards understanding and designing closed loop, real-time neurostimulation devices.
\end{abstract}

\section{INTRODUCTION}

Electrical stimulation of mammalian nerve fibers is used to treat various neurophysiological disorders. For example, deep brain stimulation of ventral intermediate nucleus is used to treat essential tremor [1]; stimulation of the vagus nerve has been effective in treating epilepsy, depression, and anxiety [2]. These nerve fibers have an ongoing physiological activity that interacts with the external current stimulus. Specifically, antidromic stimulus initiated Action Potentials (APs) may collide with orthodromic APs from ongoing physiological activity, or the nerve fiber may lose excitability or not respond to an AP (either physiological or stimulus generated) if it received a recent stimulus. In order to better understand and optimally design stimulation therapies for diseases, it is necessary to understand to what extent the physiological APs make it to the top of the fiber (i.e. to the brain) under different stimulation settings.

V. Sadashivaiah, P. Sacré, and S. V. Sarma are with the Institute for Computational Medicine and the Department of Biomedical Engineering, The Johns Hopkins University, Baltimore, MD (vjs@jhu.edu, p.sacre@jhu.edu, sree@jhu.edu).

Y. Guan is with the Department of Anesthesiology/Critical Care Medicine, The Johns Hopkins University School of Medicine, Baltimore, MD(yguan1@jhmi.edu).

W. S. Anderson is with the Institute for Computational Medicine and the Department of Neurosurgery, The Johns Hopkins University School of Medicine, Baltimore, MD (wanders5@jhmi.edu).
Most neurostimulation systems operate in an open-loop manner where the stimulation settings, like frequency, amplitude, and pattern, are preprogrammed. There is no feedback mechanism to dynamically update these parameters based on any physiological output. While the open-loop mechanism is simpler and provides short-term therapeutic benefits, it has suboptimal efficacy over long-term use. For example, open-loop spinal cord stimulation is used to treat chronic and acute pain. These systems may provide too much or too less therapy since the stimulation parameters are not based on patient's body position [3], [4]. On the other hand, closedloop neurostimulation systems can adjust their stimulation parameters on the fly and thereby provide effective and efficient therapies while also reducing the side effects of stimulation. Computational models used to design these systems can be fairly complex and thus affect their realtime performance. This calls for reduced models which can capture relatively similar information as higher-order mechanistic models but are computationally less complex.

In our previous study [5], we presented a theoretical (probabilistic) model of mammalian nerve fiber to determine how many physiological APs were relayed to the top of the fiber under different stimulation settings. We compared reliability results from the theoretical model to those generated by a detailed mechanistic model of the nerve fiber; and although the simulation runtime was very low (order of seconds) for the theoretical model, it only captured a subset of interactions and differed in results than the mechanistic model.

In this paper, we repeated our study by replacing the theoretical model with a functional model of the nerve fiber and compared relay reliability computed from this new model to that derived from the mechanistic model. The functional model is a simple model of a myelinated nerve fiber that is based on parameters like the speed of conduction, the activation threshold, and the refractory period, while the mechanistic model is a detailed higher-order model of single cable myelinated nerve fiber that is based on biophysical principles. In each model, the nerve fiber receives two inputs: the ongoing physiological activity at one end of the fiber and the external stimulus applied to the middle of the fiber. We then compute relay reliability, defined as the ratio of the number of physiological APs that make it to the other end of the fiber over the total number of physiological APs entering nerve fiber. Reliability depends on stimulus and physiological activity signal parameters including but not limited to frequency, amplitude and pattern (tonic vs bursting vs stochastic). In this study, we vary the frequency of the input signals and analyze reliability under different 
fiber diameters. Our results suggest that the functional model (runtime of the order of minutes) and the mechanistic model (runtime of the order of days) have similar reliability trends.

\section{MODEL DESCRIPTION}

\section{A. Mechanistic model of a nerve fiber}

Methods used in this section are derived from [5]. Briefly, a myelinated nerve fiber is a cylindrical active membrane (axon), tightly wrapped in an insulating myelin sheath. This myelin sheath is interrupted periodically, leaving short gaps where the axonal membrane is exposed. Following McNeal's model [6], a myelinated nerve fiber is represented by an (infinite) series of compartment elements linked by intracellular conductances. The dynamics of the membrane potential $V_{i}=V_{i}^{\text {int }}-V_{i}^{\text {ext }}$ at node $i$ (where $V_{i}^{\text {int }}$ and $V_{i}^{\text {ext }}$ are the intracellular and extracellular potentials) read as follows

$$
\begin{aligned}
C_{\mathrm{m}} \dot{V}_{i}+\sum_{k \in \mathcal{K}} I_{i, k}= & G_{\mathrm{a}}\left(V_{i-1}-2 V_{i}+V_{i+1}\right) \\
& +G_{\mathrm{a}}\left(V_{i-1}^{\mathrm{ext}}-2 V_{i}^{\mathrm{ext}}+V_{i+1}^{\mathrm{ext}}\right),
\end{aligned}
$$

where $C_{\mathrm{m}}$ is the membrane capacitance and $G_{\mathrm{a}}$ is the internodal conductance. Ionic currents $I_{i, k}$ at node $i$ include a sodium, a fast potassium, and a slow potassium ion channel, as well as a leakage current across the membrane based on the Frankenhaeuser-Huxley model [7], adjusted to experimental data of human sensory fibers at $37^{\circ} \mathrm{C}$ [8]. A complete description of the fiber model and its parameters is presented in [8], [9].

\section{B. Functional model of a nerve fiber}

In this section, we describe our functional model wherein the nerve fiber is characterized by its geometry (length and diameter) and three macroscopic properties: activation threshold, conduction velocity, and refractory period of the fiber. These macroscopic properties can be estimated either from biological experiments or from our high-dimensional mechanistic model [5]. It is assumed that:

- A stimulation or physiological input creates an action potential in the fibers if and only if its amplitude is larger than the associated threshold and its timing is not within the refractory period of a preceding action potential;

- A stimulation generated action potential propagates in the fiber in both directions (orthodromic and antidromic) at a constant velocity (the conduction velocity);

- The interaction of an orthodromic physiologicallyinduced AP and antidromic AP induced by stimulation results in a collision, i.e., the annihilation of both action potentials.

Based on these characteristics, we can identify the interactions occurring in the fiber based only on the timing of physiological and stimulation pulses [10].

\section{Electrical field potential generated by stimulation.}

The extracellular medium may be assumed to be infinite and isotropic with the electrode represented by point sources at the center $x_{j}^{\mathrm{c}}$ of each contact. Therefore, the electrical potential field at time $t$ and position $x$ is given by

$$
\varphi(t, x)=\sum_{j \in \mathcal{C}} \frac{\rho_{\mathrm{m}}}{4 \pi\left\|x-x_{j}^{\mathrm{c}}\right\|_{2}} I_{j}^{\mathrm{stim}}(t),
$$

where $I_{j}^{\text {stim }}$ is the current of point source $j$ and $\rho_{\mathrm{m}}$ is the extracellular medium resistivity. The extracellular potential at node $i$ is given by $V_{i}^{\operatorname{ext}}(t)=\varphi\left(t, x_{i}\right)$, where $x_{i}$ is the position of node $i$.

The stimulation current input $I^{\text {stim }}(t)$ consists of the repetition, at a constant frequency, $f^{\text {stim }}$, of symmetrical biphasic pulses with an amplitude ranging from $1.8 \mathrm{~mA}$ to $2.5 \mathrm{~mA}$ (increasing activation thresholds as fiber diameter increases) and a duration of $350 \mu$ s [11]. We consider stimulation frequencies ranging from 1 to $50 \mathrm{~Hz}$.

\section{Underlying physiological activity.}

The underlying physiological activity in fibers spans a broad frequency range and exhibits various patterns [12]: regular spike discharge, regular discharge of doublet spikes, bursting patterns, sporadic activity with no regular or predictable firing pattern, etc.

The presence of underlying activity in the nerve fiber is represented by replacing a 'sealed-end' boundary condition by a current source at one end of the nerve fiber. Therefore, the dynamics of the first node becomes

$C_{\mathrm{m}} \dot{V}_{1}+\sum_{k \in \mathcal{K}} I_{1, k}=G_{\mathrm{a}}\left(V_{2}-V_{1}\right)+G_{\mathrm{a}}\left(V_{2}^{\mathrm{ext}}-V_{1}^{\mathrm{ext}}\right)+I^{\mathrm{phys}}(t)$,

where the input $I^{\text {phys }}(t)$ represents the underlying activity.

The underlying physiological activity input $I^{\text {phys }}(t)$ is modeled as a Poisson train of square pulses with an amplitude of $4 \mathrm{nA}$ to $7 \mathrm{nA}$ (increasing activation thresholds as fiber diameter increases) and a duration of $1 \mathrm{~ms}$. Therefore, the instantaneous firing rate $\lambda^{\text {phys }}$ is assumed constant, ranging from $1 \mathrm{~Hz}$ to $50 \mathrm{~Hz}$, a typical range for motor and sensory firing activity [13], [14].

\section{E. Reliability}

When stimulation is applied to a nerve fiber, it ultimately is interfering with the ongoing physiological activity that travels from one end of the fiber to the other. To succinctly quantify the effects of stimulation on the fiber activity, we wanted to capture how the stimulation influences the physiologically generated APs that make it to the other end of the fiber. To quantify the effects of stimulation on the nerve fiber, we define the following reliability metric:

$$
R\left(\lambda^{\text {phys }}, f^{\text {stim }}\right)=\frac{\# \text { of relayed physiological APs }}{\text { total \# of physiological APs }},
$$

where relayed action potentials are underlying physiological inputs that travel from one end to the other end of the fiber. Metric (3) captures the effect that the stimulation has 
on the ongoing physiological activity. If $R=1$ then the stimulation has no effect, and if $R=0$, then the stimulation blocks all physiological activity from transmitting to the brain.

\section{RESULTS}

In this section, we show relay reliability results for our two models (functional and mechanistic) of a $10 \mathrm{~cm}$-long nerve fiber, that includes a monopolar electrode placed $3.5 \mathrm{~mm}$ away from the center of the fiber. We consider three different diameters of nerve fibers in our simulations $(6 \mu \mathrm{m}, 9 \mu \mathrm{m}$ and $12 \mu \mathrm{m})$. These results are drawn from 50 simulations for each frequency pair (physiological, stimulus) of this model with a stochastic Poisson physiological input. All the mechanistic model simulations were performed on NEURON simulation environment [15]. Analysis of data and functional model simulations were performed on MATLAB, MathWorks.

\section{A. Reliability: Functional vs Mechanistic model}

Physiological reliability map of the functional and mechanistic models for three different diameters $(6 \mu \mathrm{m}, 9 \mu \mathrm{m}$ and $12 \mu \mathrm{m})$ are presented in Fig. 1. We see that at low stimulation and physiological frequencies $(1 \mathrm{~Hz}$ to $10 \mathrm{~Hz})$, reliability is almost $1(100 \%)$ and there is more agreement between the two models. As the stimulus frequency increases, reliability decreases but the trend between models is similar.

\section{B. Influence of fiber diameter on reliability}

For different fiber diameters, the reliability maps change (see Fig. 1). We observe a horizontal shift in the pattern as we go from $6 \mu \mathrm{m}$ to $12 \mu \mathrm{m}$ diameter fibers. Consider a stimulus frequency of $50 \mathrm{~Hz}$ and physiological frequency of $10 \mathrm{~Hz}$, we see that the reliability values for $12 \mu \mathrm{m}$ are greater than that of $9 \mu \mathrm{m}$, which are greater than $6 \mu \mathrm{m}$. This is because of the low conduction velocity of AP's in small fibers. With an increase in fiber diameter, the reliability increases because the interaction count is reduced at higher conduction velocities. Fig. 2(b,c) shows the difference between the reliability maps of $6 \mu \mathrm{m}$ and $12 \mu \mathrm{m}$ diameter fibers for functional and mechanistic models.

\section{DiscusSION}

In this work, we studied the interactions between electrical stimulation and physiological activity induced action potentials in a mammalian nerve fiber. We found that reliability depends on stimulus frequency and physiological parameters such as frequency and diameter of the nerve fiber, both affecting the interactions between APs generated by both inputs.

\section{A. Functional versus mechanistic model}

The functional model captures many reliability properties that the mechanistic model captures, but with significantly less time and less computation power. This is because the mechanistic model involves solving multi-dimensional differential equations at each fiber node to compute signal transmission. In contrast, the functional model uses the
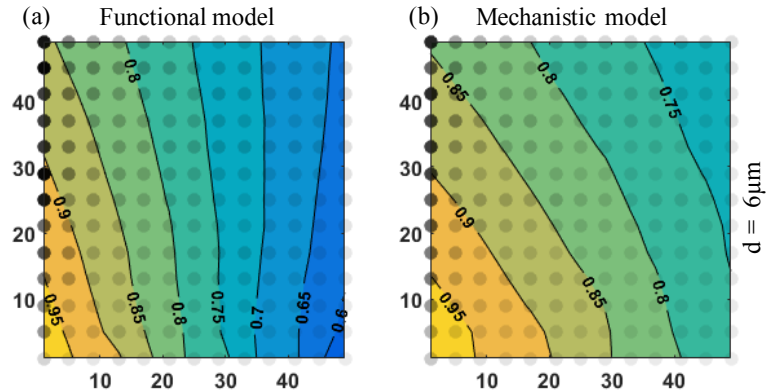

(c)

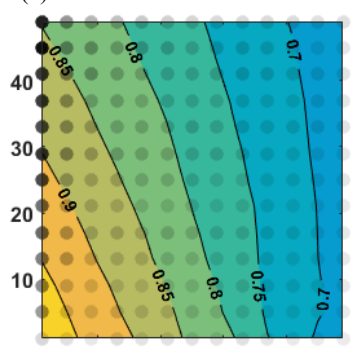

(d)
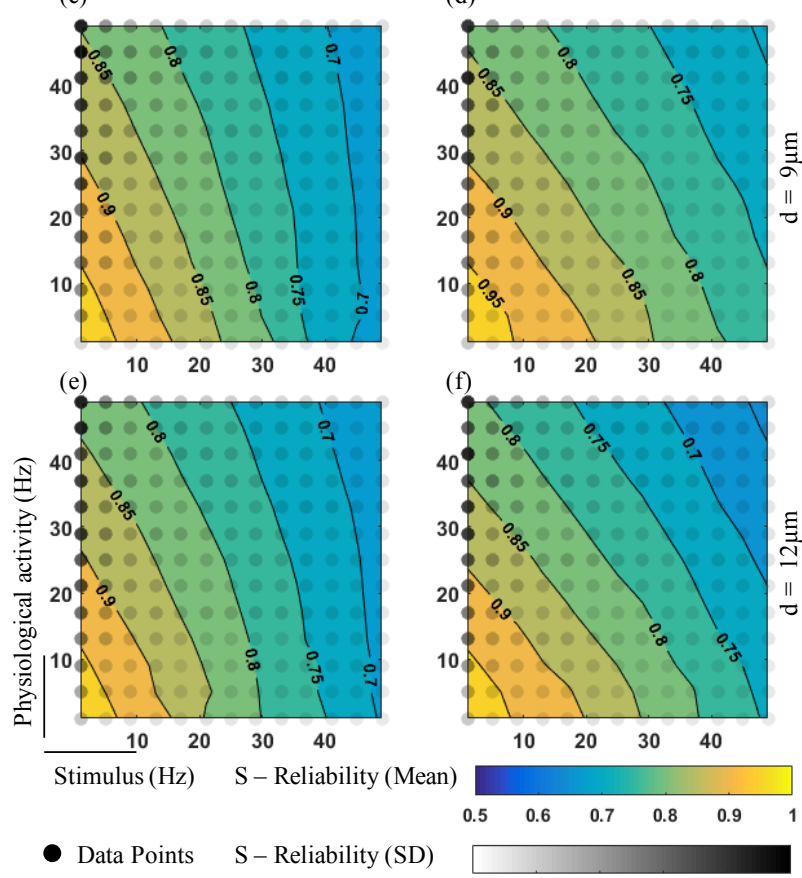

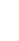

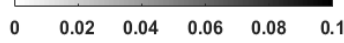

Fig. 1. Reliability maps of functional and mechanistic model for $6 \mu \mathrm{m}$, $9 \mu \mathrm{m}$ and $12 \mu \mathrm{m}$ diameters. Contour map of reliability values for a range $(1 \mathrm{~Hz}$ to $50 \mathrm{~Hz})$ of physiological frequency (Y-axis) and stimulus frequency (X-axis). Color gradient represents the mean of reliability values $(0.5-1)$. Grayscale gradient represents the standard deviation (SD) of conduction reliability values $(0-0.1)$.

activation threshold, the speed of conduction, and refractory periods to compute reliability statistics.

As expected, we found that the reliability is high at low physiological and stimulus frequencies, because fewer interactions between simulation-evoked activity and underlying physiological activity occur. As the frequencies of stimulus and physiological inputs increase, reliability decreases. This is due to the increase in the number of interactions. The difference in reliability values between the functional and mechanistic models can be attested to non-linear effects such as relative refractory period captured only by mechanistic model.

\section{B. Influence of fiber diameter on reliability.}

We see from Fig. 2(b,c), that increasing the diameter of the nerve fiber from $6 \mu \mathrm{m}$ to $12 \mu \mathrm{m}$ shifts the reliability map to the right. This is expected, since the conduction speeds are 


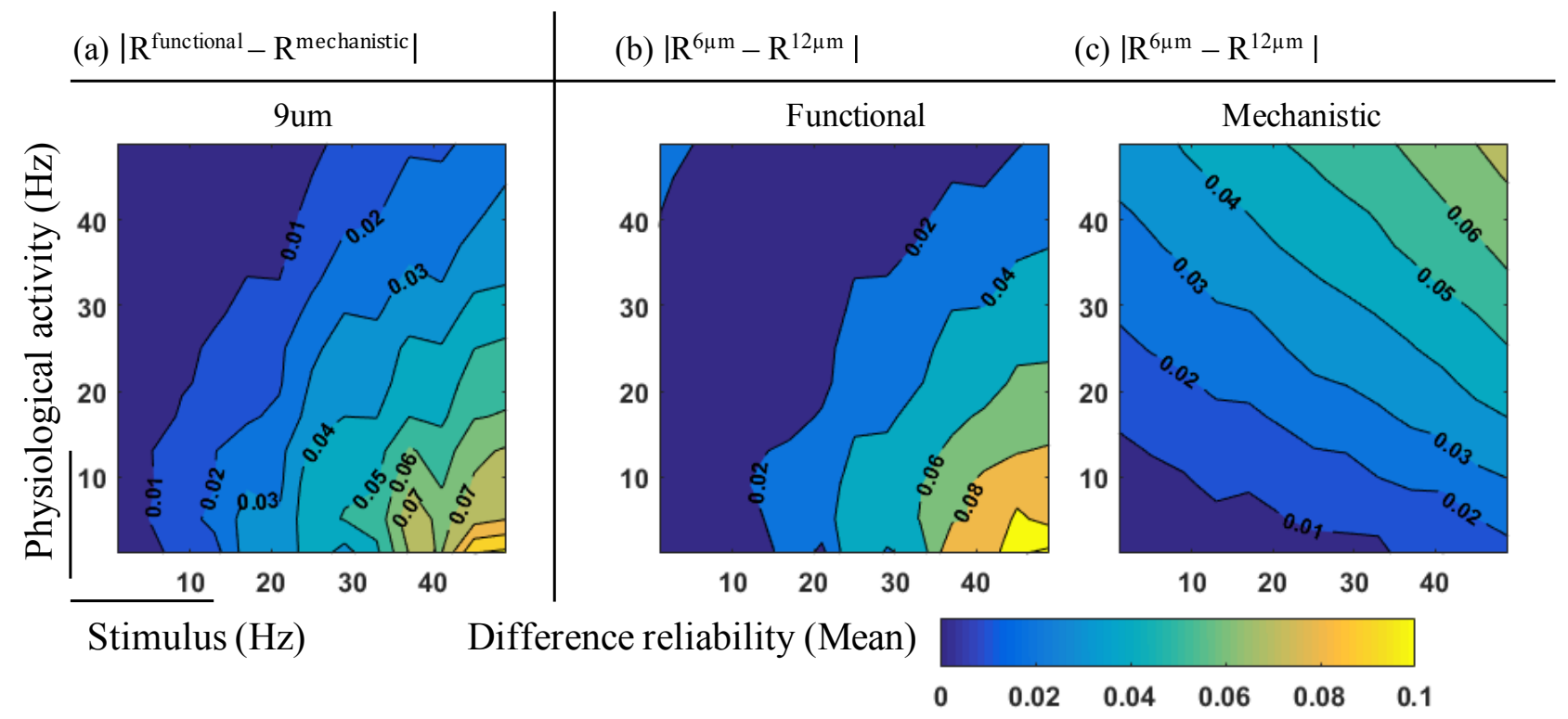

Fig. 2. Influence of diameter on reliability. a. Reliability map of abs(reduced - mechanistic) model for $9 \mu \mathrm{m}$ diameter. b,c. Reliability map of abs $(6 \mu \mathrm{m}$ vs $12 \mu \mathrm{m}$ ) diameters for functional and mechanistic models respectively.

higher in a bigger fiber [16], and therefore requires a higher frequency stimulus to achieve the relay properties observed for lower stimulus frequencies in smaller fibers.

An effective stimulation system should block the propagation of noxious (pain) information while allowing innocuous (physiological) information to reach the brain. If noxious information is modeled in a similar fashion, we can define a selective reliability metric, $R_{\text {sel }}=R_{\text {physiological }} *(1-$ $\left.R_{\text {pain }}\right)$. Our goal is to optimize stimulus parameters like frequency, amplitude to maximize $R_{\text {sel }}$.

\section{Future work}

In the future, we plan (i) to further study the interactions and quantify them statistically, and (ii) to develop a closed loop system with relay reliability fed back to optimize stimulation parameters.

\section{ACKNOWLEDGMENT}

Work supported by NIH R01 AT009401 to S.V.S., Y.G., and W.S.A, and a NPRI Postdoctoral fellowship to P.S. We would like to thank Dr. M. Caterina, Neurosurgery Pain Research Institute, The Johns Hopkins University School of Medicine, for valuable and insightful discussions.

\section{REFERENCES}

[1] K. E. LYONS and R. PAHWA, "Deep brain stimulation and essential tremor," Journal of clinical neurophysiology, vol. 21, no. 1, pp. 2-5, 2004.

[2] D. A. Groves and V. J. Brown, "Vagal nerve stimulation: a review of its applications and potential mechanisms that mediate its clinical effects," Neuroscience \& Biobehavioral Reviews, vol. 29, no. 3, pp. 493-500, 2005.

[3] G. BAROLAT, S. ZEME, and B. KETCIK, "Multifactorial analysis of epidural spinal cord stimulation," Stereotactic and functional neurosurgery, vol. 56, no. 2, pp. 77-103, 1991.
[4] J. C. Olin, D. H. Kidd, and R. B. North, "Postural changes in spinal cord stimulation perceptual thresholds," Neuromodulation: Technology at the Neural Interface, vol. 1, no. 4, pp. 171-175, 1998

[5] V. Sadashivaiah, P. Sacré, Y. Guan, W. S. Anderson, and S. V. Sarma, "Modeling electrical stimulation of mammalian nerve fibers: A mechanistic versus probabilistic approach," in 2017 39th Annual International Conference of the IEEE Engineering in Medicine and Biology Society (EMBC), July 2017, pp. 3868-3871.

[6] D. R. McNeal, "Analysis of a model for excitation of myelinated nerve," IEEE Transactions on Biomedical Engineering, vol. 23, no. 4, pp. 329-337, 1976.

[7] B. Frankenhaeuser and A. F. Huxley, "The action potential in the myelinated nerve fibre of Xenopus laevis as computed on the basis of voltage clamp data," The Journal of Physiology, vol. 171, no. 2, p. 302, 1964.

[8] J. R. Schwarz, G. Reid, and H. Bostock, "Action potentials and membrane currents in the human node of ranvier," Pflügers Archiv, vol. 430 , no. 2 , pp. 283-292, 1995.

[9] W. A. Wesselink, J. Holsheimer, and H. B. K. Boom, "A model of the electrical behaviour of myelinated sensory nerve fibres based on human data," Medical \& Biological Engineering \& Computing, vol. 37, no. 2, pp. 228-235, 1999.

[10] A. Gonzalez-Perez, R. Budvytyte, L. D. Mosgaard, S. Nissen, and T. Heimburg, "Penetration of action potentials during collision in the median and lateral giant axons of invertebrates," Physical Review X, vol. 4, no. 3, p. 031047, 2014.

[11] J. T. Mortimer and N. Bhadra, "Peripheral nerve and muscle stimulation," in Neuroprosthetics: Theory and Practice, K. W. Horch and G. S. Dhillon, Eds. World Scientific, 2004, pp. 638-682.

[12] K. C. Kajander and G. J. Bennett, "Onset of a painful peripheral neuropathy in rat: a partial and differential deafferentation and spontaneous discharge in A beta and A delta primary afferent neurons," Journal of Neurophysiology, vol. 68, no. 3, pp. 734-744, 1992.

[13] B. Katz, "Action potentials from a sensory nerve ending," The Journal of Physiology, vol. 111, no. 3-4, p. 248, 1950.

[14] C. J. De Luca, R. S. LeFever, M. P. McCue, and A. P. Xenakis, "Behaviour of human motor units in different muscles during linearly varying contractions," The Journal of Physiology, vol. 329, no. 1, pp. 113-128, 1982.

[15] M. L. Hines and N. T. Carnevale, "The NEURON simulation environment," Neural Computation, vol. 9, no. 6, pp. 1179-1209, 1997.

[16] J. B. Hursh, "Conduction velocity and diameter of nerve fibers," American Journal of Physiology, vol. 127, no. 1, pp. 131-139, May 1939. 